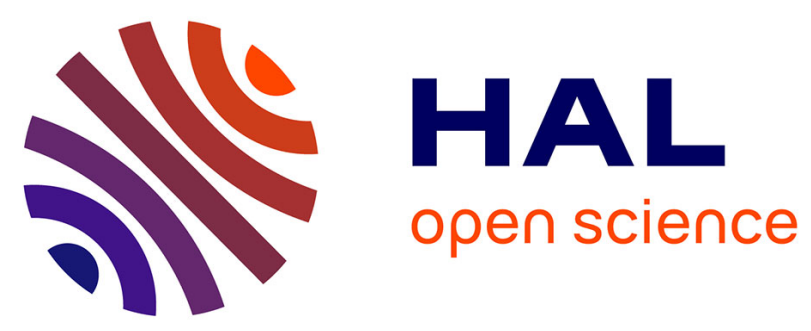

\title{
Nonlinear propagation of Rossby-Khantadze electromagnetic planetary waves in the ionospheric E-laye
}

\author{
Shimpei Futatani, Wendell Horton, Tamaz Kaladze
}

\section{To cite this version:}

Shimpei Futatani, Wendell Horton, Tamaz Kaladze. Nonlinear propagation of Rossby-Khantadze electromagnetic planetary waves in the ionospheric E-laye. Physics of Plasmas, 2013, 20, pp.102903. 10.1063/1.4826592 . hal-00931519

\section{HAL Id: hal-00931519 https://hal.science/hal-00931519}

Submitted on 11 Apr 2016

HAL is a multi-disciplinary open access archive for the deposit and dissemination of scientific research documents, whether they are published or not. The documents may come from teaching and research institutions in France or abroad, or from public or private research centers.
L'archive ouverte pluridisciplinaire HAL, est destinée au dépôt et à la diffusion de documents scientifiques de niveau recherche, publiés ou non, émanant des établissements d'enseignement et de recherche français ou étrangers, des laboratoires publics ou privés. 


\section{AIP | Physics of

\section{Nonlinear propagation of Rossby-Khantadze electromagnetic planetary waves in the ionospheric E-layer}

S. Futatani, W. Horton, and T. D. Kaladze

Citation: Physics of Plasmas 20, 102903 (2013); doi: 10.1063/1.4826592

View online: http://dx.doi.org/10.1063/1.4826592

View Table of Contents: http://scitation.aip.org/content/aip/journal/pop/20/10?ver=pdfcov

Published by the AIP Publishing

\section{Articles you may be interested in}

Effect of a novel nonlinearity, viz., electron temperature dependence of electron-ion recombination on electromagnetic wave. Plasma interaction: Nonlinear propagation in the E-layer

Phys. Plasmas 23, 032307 (2016); 10.1063/1.4943873

Rossby-Khantadze electromagnetic planetary waves driven by sheared zonal winds in the E-layer ionosphere Phys. Plasmas 22, 012906 (2015); 10.1063/1.4906362

Enhanced nonlinear interaction of powerful electromagnetic waves with ionospheric plasma near the second electron gyroharmonic

Phys. Plasmas 20, 052904 (2013); 10.1063/1.4807038

Excitation of zonal flow and magnetic field by Rossby-Khantadze electromagnetic planetary waves in the ionospheric E-layer

Phys. Plasmas 19, 022902 (2012); 10.1063/1.3681370

Generation of density irregularities and whistler waves by powerful radio waves in the polar ionosphere

Phys. Plasmas 8, 277 (2001); 10.1063/1.1334610

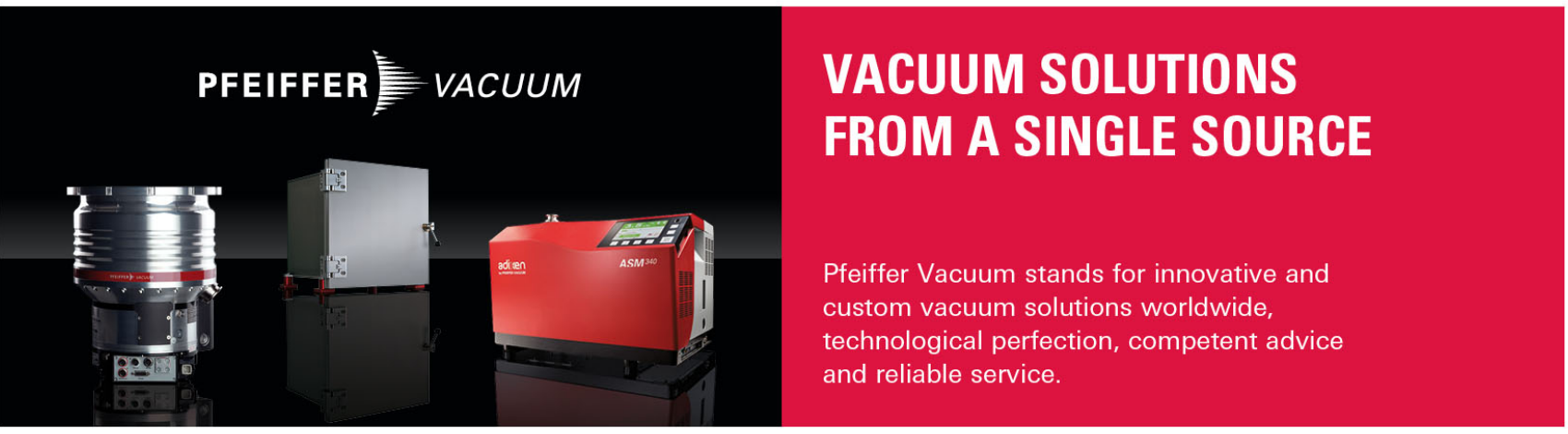




\title{
Nonlinear propagation of Rossby-Khantadze electromagnetic planetary waves in the ionospheric E-layer
}

\author{
S. Futatani, ${ }^{1}$ W. Horton, ${ }^{2}$ and T. D. Kaladze ${ }^{3,4}$ \\ ${ }^{1}$ LMFA-CNRS, École Centrale de Lyon, Université de Lyon, Ecully, France \\ ${ }^{2}$ Applied Research Laboratory, The University of Texas at Austin, Austin, Texas 78758, USA \\ ${ }^{3}$ I. Vekua Institute of Applied Mathematics, Tbilisi State University, 2 University St., 0186 Tbilisi, Georgia \\ ${ }^{4}$ Physics Department, Government College University, Lahore 54000, Pakistan
}

(Received 5 August 2013; accepted 10 October 2013; published online 28 October 2013)

Nonlinear vortex propagation of electromagnetic coupled Rossby and Khantadze planetary waves in the weakly ionized ionospheric E-layer is investigated with numerical simulations. Large scale, finite amplitude vortex structures are launched as initial conditions at low, mid, and high latitudes. For each $k$-vector the linear dispersion relation has two eigenmodes corresponding to the slow magnetized Rossby wave and the fast magnetic Khantadze wave. Both waves propagate westward with local speeds of the order of $10-20 \mathrm{~m} / \mathrm{s}$ for the slow wave and of the order of $500-1000 \mathrm{~km} / \mathrm{s}$ for the fast wave. We show that for finite amplitudes there are dipole solitary structures emitted from the initial conditions. These structures are neutrally stable, nonlinear states that avoid radiating waves by propagating faster than the corresponding linear wave speeds. The condition for these coherent structures to occur is that their amplitudes are such that the nonlinear convection around the core of the disturbance is faster than the linear wave speed for the corresponding dominant Fourier components of the initial disturbance. The presence of the solitary vortex states is indicative of an initial strong disturbance such as that from a solar storm or a tectonic plate movement. We show that for generic, large amplitude initial disturbances both slow and fast vortex structures propagate out of the initial structure. (C) 2013 AIP Publishing LLC.

[http://dx.doi.org/10.1063/1.4826592]

\section{INTRODUCTION}

In the atmosphere the large scale planetary waves and vortex structures with wavelengths $\lambda>10^{3} \mathrm{~km}$ have periods of several days and are called Rossby waves. ${ }^{1}$ At higher altitudes in the E-layer of the ionosphere the weakly ionized atmosphere is subjected to a Lorentz $\boldsymbol{j} \times \boldsymbol{B}$ force in addition to the Coriolis force owing to the magnetized electron fluid that is coupled by charge neutrality to the ion fluid which, in turn, is fixed in the neutral gas by the ion-neutral collisions. The electrodynamics of the E-layer gas modifies the Rossby wave dynamics by coupling the waves to high frequency, fast group velocity magnetic Khantadze waves. ${ }^{2}$ The partial differential equations for the coupled neutral fluid and the weakly magnetized ionized gas component are derived in Ref. 3 .

Here we present simulations showing that in the absence of meridional variations of the background gas, dipolar vortex structures propagate uniformly for many times the planetary radius. A noisy, generic large amplitude initial condition will launch both dispersive waves and faster, nonlinear dipolar vortex structures at speeds just above the linear wave speeds. Both the slow and fast structures propagate to the west. Initial perturbations with small amplitudes propagate as two types of waves: a slow dispersive wave corresponding to a Rossby wave in neutral gas of the troposphere and a fast magnetic wave with the unmagnetized ions and the inertial given by the mass density of the neutral gas. These magnetic waves in the E-layer are called Khantadze waves and propagate slowly compared to the Alfven waves in the E-layer. Thus, the E-layer has its own characteristic waves owing to the fact that the density of the ionized component is six orders of magnitude lower than that of the neutral gas density, yet the electrons are magnetized and $\boldsymbol{E} \times \boldsymbol{B}$ drift giving a strong Hall current. The ion-neutral collision time is on the order of tens of milliseconds so that in low frequency waves the ions and neutrals move together. The winds in the neutral gas component carry the ions with them which create a dynamo electric field proportional to the velocity component of the neutral gas perpendicular to the Earth's magnetic field $B_{0}$. The motion of the ionized component in the crossed electric and magnetic field generates a substantial electron current since the electrons are magnetized and $\boldsymbol{E} \times \boldsymbol{B}$ drift producing a Hall current analogous to semiconductors.

The simulations show that nonlinear initial dipolar vortex structures propagate as solitary structures while a small amplitude dipole radiates a spectrum of waves. The Lorentz force and Faraday's law couple the dynamics of the magnetic fluctuations and the stream function for the weakly ionized gas. We show that when the initial magnetic fluctuation is large, with a vanishing initial stream function a fast magnetic dipolar solitary wave is generated, and, conversely, when the initial stream function is large and magnetic perturbation is zero, a slow dipolar Rossby wave structure is generated.

The reduction of the vector equations of motion introduces the stream function $\psi$ for the horizontal flow velocity field $v$ and the magnetic flux function $A$ for the perturbation $\delta \boldsymbol{B}$ to the geomagnetic field. The coupling of the neutral gas to the plasma component is strong owing to the ion-neutral collision frequency of order $\nu_{i, n}$ being of order $100 / s$. The coupled dynamics gives rise to the two types of low 
frequency waves with their associated nonlinear vortex structures from the Jacobian, or Poisson bracket, nonlinearities intrinsic to the convective derivatives. Both structures are of interest because of their significant influence on the global atmospheric circulation and the ultra-low frequency magnetic signals emitted by the oscillating electron Hall currents produced by the $\boldsymbol{E} \times \boldsymbol{B}$ motion of the electrons in the E-layer. The magnetic signals are readily detected and are of practical importance for the early detection of large scale disturbances to the upper atmosphere and the ionosphere.

There is observational data that verifies the presence of ultra low-frequency (ULF) electromagnetic (EM) planetary scale perturbations in the ionosphere.$^{6-10}$ Large amplitude ionospheric disturbances are produced by phenomena like earthquakes, volcano eruptions, and the ionospheric response to anthropogenic activity. Recent observations show that forced oscillations of that kind appear in impulsive impacts on the ionosphere from rocket launches, explosions or from magnetospheric compressions during severe magnetospheric storms.

The study of the generation and the dynamics of planetary Rossby waves that are induced by the spatial inhomogeneity of the Earth's angular velocity in the ionospheric plasma has accordingly been a subject of a great deal of theoretical and experimental investigations in recent years. ${ }^{4,5}$ The presence of charged particles from the low density $n_{e}$ plasma component in the lower ionosphere adds a new branch of low-frequency waves in the thermosphere-ionosphere system.

In Sec. II we give the nonlinear partial differential equations of the coupled dynamics of the neutral gas and the plasma component of the lower ionosphere. The coupling gives rise to a transfer of energy between the neutral and ionized components. In Sec. III we show the linear dispersion relation for small amplitude waves and define the slow Rossby wave and the fast magnetic wave called the Khantadze wave in the literature. In Sec. IV we solve the coupled partial differential equations with numerical simulations. The simulations show that for finite amplitude waves nonlinear vortex structures are formed and propagate as nonlinear vortex structures. In Sec. IV comparisons are made between the initial perturbations that have principally neutral gas flows and those with principally electromagnetic perturbations. The study gives the results of large scale simulations for dipoles launched at low latitude, midlatitude, and high latitude. In Sec. V the conclusions are given.

\section{MODELING FOR THE LOW FREQUENCY STRUCTURES IN E-LAYER IONOSPHERE}

Large scale, fast waves are generated when the latitudinal gradient of vertical component of geomagnetic field, and the Hall effect is taken into account in the dynamical equations. The north-south convection of the fluid introduces a parameter $\beta_{B}$ measuring the change of the vertical geomagnetic field with latitude $\lambda$ as a horizontal element of fluid $d x d y$ moves in the vortex. The curl of the electric field generated by $\boldsymbol{v} \times\left(\boldsymbol{B}_{0 z}+\delta \boldsymbol{B}_{z}\right)$ gives a contribution from $\beta_{B}$ $=\frac{\partial B_{0 z}}{\partial y}=-2 B_{e q} \cos \left(\lambda_{0}\right) / R$, and the magnetic perturbation $\delta B_{z}(x, y, t)$ is then transported by Faraday and Ampere's law to move with velocity $c_{B}=\frac{\beta_{B}}{e n_{e} \mu_{0}}$, where $n_{e}$ is the electron number density and $B_{e q}$ is the equatorial value of the geomagnetic field at a distance $R$ from the Earth's center. The linear part of the fast wave is a magnetic perturbation propagating as $\delta B_{z}\left(x-c_{B} t\right)$, where $c_{B}$ is of order $500 \mathrm{~m} / \mathrm{s}$ on the day side to $5 \mathrm{~km} / \mathrm{s}$ on the nightside owing to the decrease in the electron density in the night time ionosphere. The derivation of the nonlinear equations for the stream function for the neutral fluid velocity measured with respect to the surface of the Earth and the coupled magnetic perturbation $\delta B_{z}$ are well known and not repeated here. ${ }^{2,3}$

The E-layer is weakly ionized with the electron and ion density $n_{e}=n_{i}$ orders of magnitude lower than the density $N$ of the neutral gas component. The E-layer gas satisfies the condition $n_{e} / N \ll 1$. At temperatures of $T_{e}=T_{i}<600 \mathrm{~K}$ the friction between the ions and neutrals dominates the ion acceleration while the electron fluid force balance is dominated by the electric field and the Lorentz force. Since the ions and neutrals are tied together on a time scale of $10 \mathrm{~ms}$, the equations of motion reduce to that of the acceleration of the neutral gas with a contribution from the $\boldsymbol{j} \times \boldsymbol{B}$ force that arises from the plasma-ion force arising from the ion-neutral drag force. The explicit electric field is eliminated in the neutral gas component when the electron and ion momentum balance equations are added to the system and quasineutrality is used. The acceleration of such a weakly ionized gas is determined by the inertia of the massive neutral gas component that responds to the Corilois force for large scale structures and the Lorentz force from the Hall current density $\boldsymbol{j}=-e n_{e}\left(\boldsymbol{v}_{e}-\boldsymbol{v}\right)$ in the nonuniform geomagnetic field. The derivation of the reduced two-dimensional motion in the tangent plane to the Earth's surface or beta-plane of geophysics describes how the forces vary with latitude $\lambda$ from the vertical components of the Earth's rotation vector $\boldsymbol{\Omega}$ and the vertical component of the local geomagnetic magnetic field $\boldsymbol{B}_{0}$. These latitudinal gradients are specified in the model by the values of $\beta$ and $\beta_{B}$. These gradient parameters determine the westward speeds of the two modes of the system.

For the typical ionization fractions of $10^{-6}$ in the E-layer, the Lorentz force is comparable to the Coriolis force. Hence, the effects of the spatially inhomogeneous geomagnetic field $\boldsymbol{B}(x)$ and the vertical component of the Earth's rotation $\Omega$, both of which are inherent in the case of EM planetary waves and must be taken into account. The ionospheric plasma is immersed in a dipole magnetic field $\boldsymbol{B}_{0}$, and we analyze the wave motions in the E-layer localized in the vicinity of a given latitude $\lambda=\lambda_{0}$. We introduce a local Cartesian system of coordinates $(x, y, z)$ with latitude $y=\left(\lambda-\lambda_{0}\right) R$ and longitude $x=\varphi R \cos \lambda_{0}$, where $R$ is the Earth's radius. The $z$-axis of the coordinate system coincides with the local vertical direction. Furthermore, $\varphi$ is the longitude, and in this coordinate system, the $x$-axis is directed from the west to the east, and the $y$-axis is directed from the south to the north. The corresponding derivatives with respect to the horizontal coordinates are given by $\partial / \partial \varphi=R \cos \lambda_{0} \partial / \partial x$ and $\partial / \partial \lambda$ $=R \partial / \partial y$. The magnitude of the dipole geomagnetic field is defined by $B_{0}=B_{e q}\left(1+3 \sin ^{2} \lambda\right)^{1 / 2}$, and the local components of the geomagnetic field vector is $\boldsymbol{B}_{0}=\left(0, B_{0 y}, B_{0 z}\right)$ $=\left(0, B_{e q} \cos \lambda,-2 B_{e q} \sin \lambda\right)$. For the angular velocity of the Earth's rotation $\boldsymbol{\Omega}$ in the local system of coordinates, we have $\boldsymbol{\Omega}=\left(0, \Omega_{0 y}, \Omega_{0 z}\right)=\left(0, \Omega_{0} \cos \lambda, \Omega_{0} \sin \lambda\right)$. 
In the E-layer, the wave motions are basically twodimensional, i.e., $\boldsymbol{v}=(u, v, 0)$, with $u=-\partial \psi / \partial y$ and $v=\partial \psi / \partial x$, where $\psi$ is the stream function. Furthermore, we will neglect the $z$-dependence of the perturbations. By means of complex calculations, one finds that we can keep the $\partial / \partial z$ derivatives, integrate over the conductive slab using the proper boundary conditions at the Earth's surface and at the magnetosphere and introduce the height integrated Hall conductivity. The results, however, are the same as the simple derivation with the constants modified by factors of order unity. Thus, to a reasonable approximation, only the $z$-component of the vorticity $\xi_{z}=\Delta \psi$ is significant for the problem. Analysis of Ref. 5 shows that only the z-component of the magnetic induction perturbation is significant, i.e., $\delta B_{z}(x, y, z)=b$. Then, from Eq. (3), we get the RossbyKhantadze waves equations which are derived in Ref. 11

$$
\begin{gathered}
\frac{\partial \Delta \psi}{\partial t}+\beta \frac{\partial \psi}{\partial x}-\beta_{A} \beta_{B} \frac{\partial b}{\partial x}=[\Delta \psi, \psi], \\
\frac{\partial b}{\partial t}+\beta_{B} \frac{\partial \psi}{\partial x}+c_{B} \frac{\partial b}{\partial x}=[b, \psi] .
\end{gathered}
$$

Here, $\psi$ is the stream function for the velocity of the neutral gas with the ionized component tied to the neutral gas. The magnetic induction perturbation in $\mathrm{z}$-component is $b=\delta B_{z}(x, y, t), \beta_{A}=1 /\left(\mu_{0} \rho\right)$, where $\mu_{0}$ is the magnetic permeability and $\rho$ is the mass density, $[a, b]=\partial_{x} a \partial_{y} b$ $-\partial_{y} a \partial_{x} b$ which is Poisson's brackets, and the two dimensional Laplacian is $\Delta=\partial_{x}^{2}+\partial_{y}^{2}$. In the vicinity of the latitude $\lambda=\lambda_{0}$, we use the $\beta$-plane approximation ${ }^{4}$ and represent the Coriolis parameter as

$$
f=2 \Omega_{0 z}=2 \Omega_{0} \sin \lambda=f_{0}+\beta y,
$$

with

$$
\beta=\frac{\partial f}{\partial y}=\frac{2 \Omega_{0} \cos \lambda_{0}}{R}>0 .
$$

Analogously, for the geomagnetic field, we represent

$$
B_{0 z}=-2 B_{e q} \sin \lambda=\gamma_{0}+\beta_{B} y,
$$

with

$$
\beta_{B}=\frac{\partial B_{0 z}}{\partial y}=-\frac{2 B_{e q}}{R} \cos \lambda_{0}<0
$$

Thus, these two coupled nonlinear partial differential equations describe the nonlinear dynamics of the electromagnetic planetary low-frequency wave perturbations in the ionospheric E-Layer. There are two energy components in the system: kinetic energy of the massive neutral fluid and magnetic energy from the currents created by the electron motion. From Eqs. (1) and (2) the total energy is the sum given by

$$
E=\frac{1}{2}\left(\int \rho|\nabla \psi|^{2} d V+\frac{1}{\mu_{0}} \int b^{2} d V\right),
$$

where the first term is the kinetic energy in the motion of the neutral atmosphere and the second term is the energy in the fluctuating magnetic field.

\section{LINEAR ANALYSIS OF ROSSBY-KHANTADZE WAVES IN THE IONOSPHERIC E-LAYER}

From Eqs. (1) and (2), the dispersion relation for the linear electromagnetic planetary waves is given by

$$
\left(\omega k_{\perp}^{2}+\beta k_{x}\right)\left(\omega+k_{x}\left|c_{B}\right|\right)-\beta_{A}\left|\beta_{B}\right|^{2} k_{x}^{2}=0,
$$

where $\omega$ is the wave frequency and $k_{\perp}^{2}=k_{x}^{2}+k_{y}^{2}, k_{x}$ and $k_{y}$ are components of the wave vector $\boldsymbol{k}$ along the $\mathrm{x}$ - and $\mathrm{y}$-axes

$$
\omega_{ \pm}=-\frac{k_{x}}{2 k_{\perp}^{2}}\left[\left|c_{B}\right| k_{\perp}^{2}+\beta \pm \sqrt{\left(\left|c_{B}\right| k_{\perp}^{2}-\beta\right)^{2}+4 k_{\perp}^{2} \beta_{A}\left|\beta_{B}\right|^{2}}\right] .
$$

The + root is the fast Khantadze wave and the - root is the slow magnetized Rossby wave root. The speeds of the two waves are pushed apart from the coupling of the flow velocity and the magnetic force. Evaluating the eigenvectors for the + and - roots we find the ratio of the flow kinetic energy to the magnetic energy in the two roots. The waves are coupled by the nonlinear interactions as shown in the simulations. The parameter $\beta_{A}\left|\beta_{B}\right|^{2}$ that arises from the latitudinal gradient of the geomagnetic field causes the coupling of two waves: the Khantadze's waves with $\omega_{+}=k_{x} c_{B}$ (fast waves) and ordinary Rossby waves with $\omega_{-}=-k_{x} \beta / k_{\perp}^{2}$ (slow waves).

The EM Rossby waves are studied for the latitude of $\lambda=45^{\circ}$ which, for reference, is the latitude of ProvenceAlpes-Côte d'Azur in France. In this work, the neutral density $N$ is fixed as $N=10^{19}\left[\mathrm{~m}^{-3}\right]$. The values of coefficients of linear terms of Eqs. (1) and (2) will be given as the second column of Table I. Figure 1 shows the dispersion relation of Eqs. (1) and (2). Khantadze wave $\left(\omega_{+}\right)$shows the strong variation in $k_{x}$. The system of the electromagnetic component for the

\begin{tabular}{|c|c|c|}
\hline & $\begin{array}{c}\lambda=45\left[^{\circ}\right] \\
n_{e}=10^{11}\left[\mathrm{~m}^{-3}\right] \\
N=10^{19}\left[\mathrm{~m}^{-3}\right]\end{array}$ & $\begin{array}{c}\lambda=45\left[^{\circ}\right] \\
n_{e}=10^{10}\left[\mathrm{~m}^{-3}\right] \\
N=10^{19}\left[\mathrm{~m}^{-3}\right]\end{array}$ \\
\hline $\begin{array}{l}\text { Mass density: } \\
\rho=N \cdot 25 m_{p}\left[\mathrm{~kg} \cdot \mathrm{m}^{-3}\right]\end{array}$ & $\rho=4.175 \times 10^{-7}$ & $\rho=4.175 \times 10^{-7}$ \\
\hline $\begin{array}{l}\text { Gradient of Coriolis force: } \\
\beta=2 \Omega_{0} \cos (\lambda) / R\left[\mathrm{~m}^{-1} \mathrm{~s}^{-1}\right]\end{array}$ & $\beta=1.1 \times 10^{-11}$ & $\beta=1.105 \times 10^{-11}$ \\
\hline $\begin{array}{l}\text { Geomagnetic field: } \\
\beta_{B}=-2 B_{e q} \cos (\lambda) / R[\mathrm{~T} / \mathrm{m}]\end{array}$ & $\beta_{B}=-1.1 \times 10^{-11}$ & $\beta_{B}=-1.105 \times 10^{-11}$ \\
\hline $\begin{array}{l}\text { Rossby speed: } \\
c_{B}=\beta_{B} /\left(e n \mu_{0}\right)[\mathrm{m} / \mathrm{s}]\end{array}$ & $c_{B}=-549$ & $c_{B}=-5500$ \\
\hline
\end{tabular}
fast wave is anisotropic in $\mathrm{x}$-direction (west-east direction) with large frequency $\omega_{+} \sim 1 \times 10^{-2}[\mathrm{rad} / \mathrm{s}]$. Rossby wave $\left(\omega_{-}\right)$gives the localized low frequencies in the vicinity of $k_{x}=k_{y}=0$ of the order of $\omega_{-} \sim-5 \times 10^{-6}[\mathrm{rad} / \mathrm{s}]$. The localized frequency of $\omega_{-}$in the vicinity of $k_{x}=k_{y}=0$ in dispersion relation which exhibits large scale dynamics interacts

TABLE I. The reference coefficients of linear terms in Eqs. (1) and (2) at mid-latitudes. 

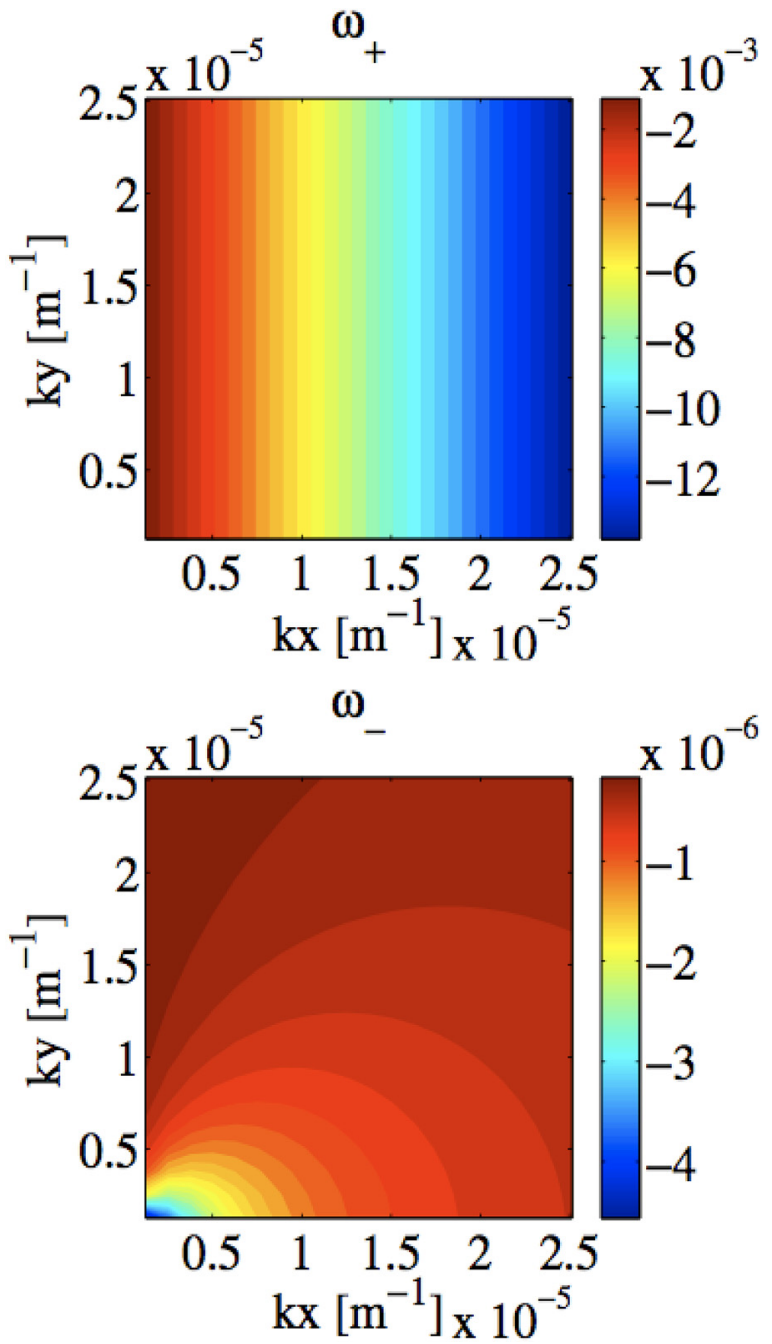

FIG. 1. Dispersion relations at the latitude of $\lambda=45^{\circ}$. The dispersion relation has two solutions for the frequency of the system. Khantadze wave $\left(\omega_{+}\left(k_{x}, k_{y}\right)\right)$ has a strong variation in $k_{x}$. The system of the electromagnetic Khantadze waves has anisotropy in x-direction (west-east direction). The Rossby waves $\left(\omega_{-}\left(k_{x}, k_{y}\right)\right)$ function is localized in the vicinity of the small $k_{x}, k_{y}$ in $\mathrm{k}$-space where the frequencies and phase velocities are low with $\omega_{-} \sim-5 \times 10^{-6}[\mathrm{rad} / \mathrm{s}]$. The dispersion relation suggests the presence of large scale dynamics which interacts with the zonal flows. For $k_{x}>0$, both frequencies, $\omega_{+}$and $\omega_{-}$, are negative which indicates the wave propagation is directed from east to west.

with the zonal flows. Figure 1 shows that there is a group velocity in $y$-direction in the Rossby wave $\left(\omega_{-}\right)$which dominates the dynamics of the stream function and the vorticity. However, there is another strong wave, Khantadze wave $\left(\omega_{+}\right)$ which dominates the electromagnetic component, is directed to the west strongly. The linear analysis indicates that the structure of the magnetic perturbation propagates to the westward strongly while the structure of the stream function moves imperceptibly.

\section{NONLINEAR SIMULATION RESULTS OF THE MAGNETIZED ROSSBY AND KHANTADZE WAVES IN THE IONOSPHERIC E-LAYER}

We leave Eqs. (1) and (2) in dimensional form using MKS units. Equation (1) has dimensions of $\left[\mathrm{s}^{-2}\right]$, and Eq. (2) has dimensions of $\left[T \mathrm{~s}^{-1}\right]$. For the ionospheric E-layer, the
TABLE II. The reference values. The order of the magnetic fluctuations is $b \sim 10^{-6}[\mathrm{~T}]$ and the order of the stream function is $\psi \sim 10^{7}\left[\mathrm{~m}^{2} / \mathrm{s}\right]$. We note that it is useful to use $[\mathrm{T}]=\left[\mathrm{kgs}^{-2} \mathrm{~A}^{-1}\right]$ and $[\mathrm{H} / \mathrm{m}]=\left[\mathrm{kgms}^{-2} \mathrm{~A}^{-2}\right]$.

Earth's radius

$$
R=6.4 \times 10^{6}[\mathrm{~m}]
$$

Rossby radius

Earth's angular velocity

$$
r_{R}=10^{6}[\mathrm{~m}]
$$

Proton mass

Electron charge

Magnetic field

$\Omega_{0}=0.5 \times 10^{-4}[\mathrm{rad} / \mathrm{s}]$

$m_{p}=1.67 \times 10^{-27}[\mathrm{~kg}]$

$e=1.602 \times 10^{-19}[\mathrm{C}]$

$B_{e q}=0.5 \times 10^{-4}[\mathrm{~T}]$

Magnetic permittivity

$\mu_{0}=1.257 \times 10^{-6}[\mathrm{H} / \mathrm{m}]$

reference values are given by Table II. We note that it is useful to use $[\mathrm{T}]=\left[\mathrm{kgs}^{-2} \mathrm{~A}^{-1}\right]$ and $[\mathrm{H} / \mathrm{m}]=\left[\mathrm{kgms}^{-2} \mathrm{~A}^{-2}\right]$.

Simulations are performed with the use of a double periodic (in $x$ - and $y$-components) Cartesian domain with a $1024 \times 1024$ grid spatial resolution. The simulation box size is $L_{x}=L_{y}=5000 \mathrm{~km}$ for the large dipole structure study, as a reference case. A finite difference method is used in which the nonlinear terms are computed using a method developed by Arakawa. ${ }^{14}$ The time stepping is performed using a predictor-corrector scheme. We use a generic dipole structure function for the initial condition of the plasma stream function, $\psi$, and magnetic fluctuations, $b$,

$$
\begin{aligned}
\psi_{t=0}= & \exp \left[\frac{\left.-\left(\left(x-x_{0}\right)\right)^{2}+\left(\left(y-y_{0}\right)+y_{d}\right)^{2}\right)}{2 \sigma^{2}}\right] \\
& -\exp \left[\frac{\left.-\left(\left(x-x_{0}\right)\right)^{2}+\left(\left(y-y_{0}\right)-y_{d}\right)^{2}\right)}{2 \sigma^{2}}\right]
\end{aligned}
$$

The position of the dipole structures is defined by $x_{0}=y_{0}$ $=2500[\mathrm{~km}] . y_{d}=0.0035 \times L_{y}[\mathrm{~km}]$ is the distance between the dipole, and the radius of the initial vortex $\sigma=2.5$ $\times 10^{5}[\mathrm{~m}]$. The same initial dipole structures are given to the magnetic fluctuations with the amplitude of $b \sim 10^{-6}[T]$, while to the stream function with the amplitude of $\psi$ $\sim 10^{7}\left[\mathrm{~m}^{2} / \mathrm{s}\right]$ as a reference value of the amplitude of the initial dipole structure. The dipole structure traps particles which remain frozen in by the magnetic field lines.

The most commonly seen vortex structures are the monopole and dipole vortices. The monopole vortex represents a net excess of mass in the vortex and radiates a wake field. Here we give preference to the dipole vortex structure which has a shift in the mass density between the lobes in dipole structure with no net change in the integrated mass density. Introducing a background shear flow complicates the problem considerably and will be considered in a subsequent work. Nonlinearity of the Rossby waves gives rise to the formation of a solitary two-dimensional coherent vortex structure, solitary localized in space as described in Ref. 12 and Horton and Hasegawa. ${ }^{13}$ Two important physical properties of solitary Rossby waves are that (i) they propagate with speeds comparable to the speeds of the linear modes and (ii) they posses a high degree of stability to perturbations.

\section{A. Break down of the solitary vortical structures in electromagnetic Rossby-Khantadze waves}

The ratio of the nonlinear frequency given by vortex rotation rate $\Omega_{E}$ to the linear frequency $\omega_{k}$ is defined as the 
$\boldsymbol{E} \times \boldsymbol{B}$ rotation number $R_{E}$ and is given by $R_{E}=\Omega_{E}(k) / \omega_{k}$. Since $R_{E}$ is the number of rotations that an $\boldsymbol{E} \times \boldsymbol{B}$ drifting particle makes in the wave period $T_{k}=2 \pi / \omega_{k}$, the value of $R_{E}$ is a Galilean invariant when $\omega_{k}$ is taken in the plasma rest frame. When $R_{E}<1$, the dispersion of the wave packet dominates and only when $R_{E} \geq 1$ the rotation of vortex structures traps the waves. This condition is the "wave trapping condition." In the first case, the dipole vortex which is the solution of the nonlinear system is initialized in order to make sure of the facts of "wave trapping condition." For small amplitudes below the wave trapping condition the dipole vortex is radiated away into many small waves. In contrast, for initial amplitudes such that the rotation frequency around the vortex structure is faster than the wave dispersion time for the vortex structure of size $\sigma$, the initial structure in Eqs. (1) and (2) is kept but for many wave periods with $k \sim 1 / \sigma$.

Figure 2 shows the stream function, the vorticity, and the magnetic fluctuations at $\mathrm{t}=600 \mathrm{~h}$. Note that the initial amplitude is small, the stream function is $\psi_{t=0} \sim 1.0$ and the magnetic fluctuation is $b_{t=0} \sim 10^{-13}$. The first row is the full system of Eqs. (1) and (2). The second row of Fig. 2 is the typical Rossby-wave which is reduced from the Eqs. (1) and (2) by switching off the magnetic fluctuations, i.e., $\beta_{B}=c_{B}=0$. In the third row of Fig. 5, the nonlinear terms from Eqs. (1) and (2) have been switched off in order to check the effect of linear terms. The structures of the stream function (the left column) show the character of linear regime, the radiative dispersion of the initial dipole structure while the structure of the magnetic fluctuation maintains its initial shape. In all three cases, even when the nonlinear terms are on, the structures of the stream function show the character of the linear regime with the radiative dispersion of the coherent structure into many elongated structures in the east-west direction. The small scale structures $(\sim 100 \mathrm{~km})$ have been also investigated and we have observed that they cannot keep the vortical shape because the rotation frequency is not faster than the wave dispersion frequency. Concerning the structure of magnetic perturbations (the right column), the first row (full system of Eqs. (1) and (2)), and the third row (without nonlinear terms) of Fig. 2 show the
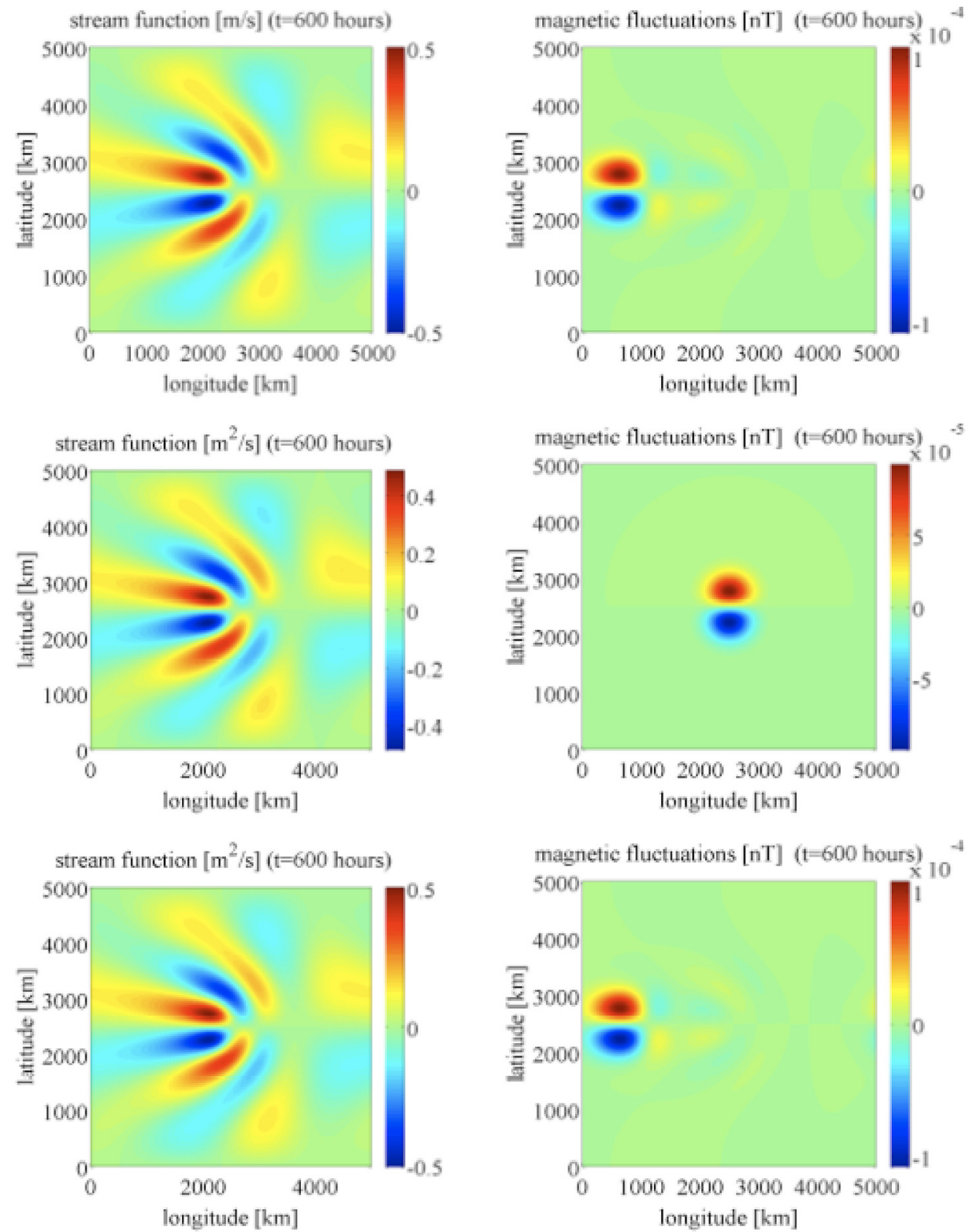
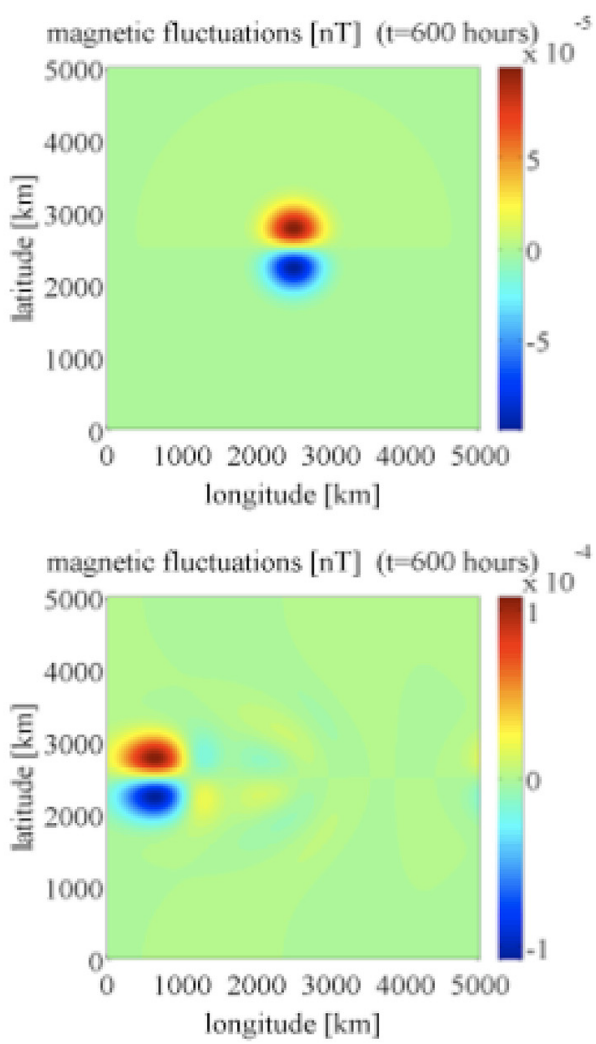

FIG. 2. The stream function and the magnetic fluctuations at $\mathrm{t}=600 \mathrm{~h}$. Initial amplitude of the stream function is $\psi_{t=0} \sim 1.0\left[\mathrm{~m}^{2} / \mathrm{s}\right]$ and the magnetic fluctuation is $b_{t=0} \sim 10^{-13}[\mathrm{~T}]$. The initial amplitude is small, and the equations are essentially linear, so there is dispersion if the vorticity equation produces a spectrum of waves while the magnetic perturbation has weak dispersion that comes from the coupling term of $\beta_{B}$ in Eq. (2). The second row of Fig. 2 shows that the classic linear Rossby wave propagates without a magnetic perturbation when the magnetic fluctuation is switched off, i.e., $\beta_{B}=c_{B}=0$. In the third row of Fig. 5 , both the nonlinear terms from Eqs. (1) and (2) have been switched off. In all cases, even if the nonlinear terms are on, the structures of the stream function show the character of the linear regime with the radiative dispersion of the coherent structure into many elongated structures in east-west direction. 
propagation of the structure to the west direction with the velocity of $c_{B}$ regardless of existence of nonlinear terms. The structure of the magnetic perturbation which is shown in second row of Fig. 2 does not move since the velocity $c_{B}$ is put zero.

\section{B. Nonlinear propagation of solitary vortical structures in electromagnetic Rossby waves}

The large amplitude of the dipole structure has the strength to maintain its shape by trapping the waves in the rotating structure, i.e., the disturbance propagates with a solitary wave character. In order to study the nonlinear propagation of solitary vortical structures, the magnetic fluctuations are initialized by giving them a dipolar structure with an amplitude of $b \sim 10^{-6}[T]$, while the stream function perturbations are given an initial amplitude of $\psi \sim 10^{7}\left[\mathrm{~m}^{2} / \mathrm{s}\right]$.

Figure 3 shows the snapshot of the stream function (left panels) and the magnetic fluctuations (right panels) at $t=0 \mathrm{~min}$ (top panels) and at $t=30 \mathrm{~min}$ (bottom panels). The dipole structure of the stream function moves imperceptibly during this time interval, while the dipole structure of the magnetic fluctuations moves to the west with the speed of $556 \mathrm{~m} / \mathrm{s}(2000 \mathrm{~km} / \mathrm{h})$. Secondary dipole magnetic structure which is linear perturbations of the magnetic field is induced by the stream function. The stream function and the magnetic perturbation interact each other. However, the influence rate between mutual physical quantities is different. The stream function carries a lot of influence on magnetic perturbation but the converse is not true. Because the stream function drives the system through the vorticity, furthermore the magnitude of the stream function and the one of magnetic perturbation are different. Therefore, the induction of the stream function by the magnetic perturbation is feeble and not visible in the stream function as the dipole structure is much larger. There is a standard Rossby wave here with small magnetic fluctuations.

In the region of India which is at the latitude of $\lambda=10^{\circ}$, the parameters of $(\lambda, n, N)$ are $\left(\lambda=10\left[^{\circ}\right], n=10^{11}, N=10^{19}\right)$ have been studied, with the values given in the third column of Table I. The Coriolis force is $2 v \times \Omega=0$ at the Equator $\sim 2 v \Omega \sin \lambda$ and so is the gradient of the Lorentz force at $\lambda=10^{\circ}$ which is higher than the case of $\lambda=45^{\circ}$. One needs a vertical component of the vectors $\Omega$ and $B$ but they are purely horizontal at the equator. The dispersion relation has two solutions for the frequency of the system. $\omega_{-}$gives the localized value in the vicinity of $k_{x}=k_{y}=0$ in the order of $\omega_{-} \sim 7 \times 10^{-6}[\mathrm{rad} / \mathrm{s}]$. The amplitude of the frequency $\omega_{-}$ is higher than the mid-latitude case. This follows from the observation that the large $\beta$ which is related to the larger gradient of the Coriolis force. The nonlinear simulation shows the movement of the dipole structures of the magnetic fluctuation to be similar to the mid-latitude case in Fig. 3. The structure of the stream function moves imperceptibly while the structure of the magnetic fluctuations moves to the west with the speed of $764 \mathrm{~m} / \mathrm{s} \quad(2750 \mathrm{~km} / \mathrm{h})$. The value of the Rossby speed is close to the one which is obtained by the linear system as given by Table I. The parameters of
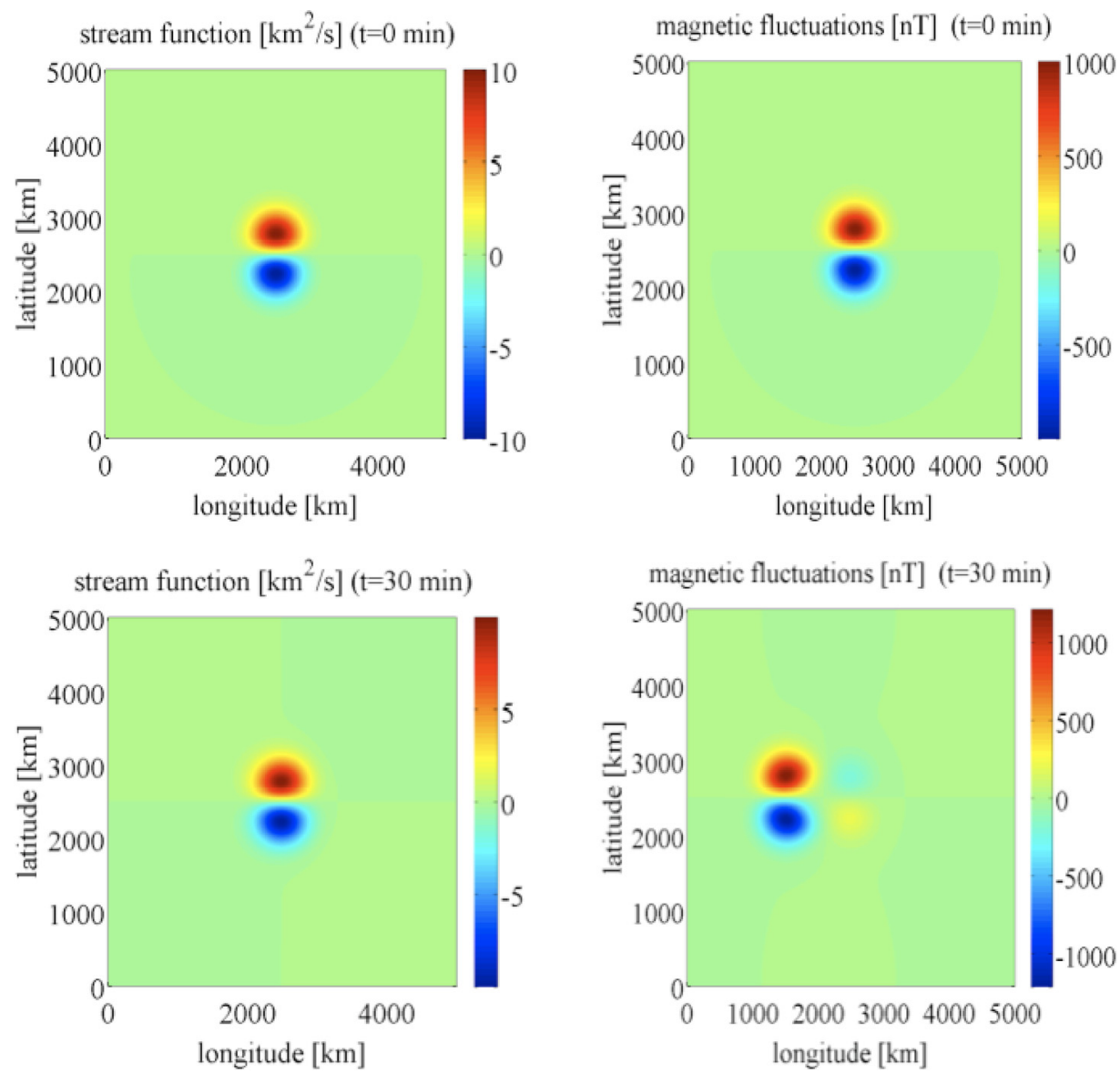

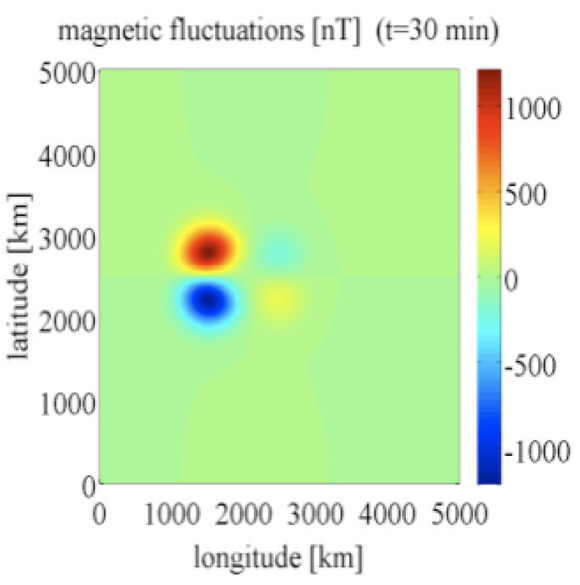

FIG. 3. $(\lambda, n, N)=\left(\lambda=45^{\circ}\right], n=10^{11}$, $\left.N=10^{19}\right)$. Snapshots of the stream function (left column) and the magnetic fluctuations (right column) at the initial condition (top rows) and $30 \mathrm{~min}$ (bottom rows). The dipole structure of the stream function moves less than $1 \mathrm{~km}$ while the dipole structure of the magnetic fluctuation moves to the west with a speed of approximately $556 \mathrm{~m} / \mathrm{s}$ $(2000 \mathrm{~km} / \mathrm{h})$. The solitary wave of the magnetic fluctuation propagates with speeds comparable to the speed $c_{B}$ of the linear modes which are given in Table I. 
TABLE III. The reference coefficients of linear terms of Eqs. (1) and (2) at high and low latitudes.

\begin{tabular}{lcc}
\hline \hline & \multicolumn{1}{c}{$\left.\lambda=800^{\circ}\right]$} & $\left.\lambda=10^{\circ}\right]$ \\
& $n_{e}=10^{11}\left[\mathrm{~m}^{-3}\right]$, & $n_{e}=10^{11}\left[\mathrm{~m}^{-3}\right]$, \\
$N=10^{19}\left[\mathrm{~m}^{-3}\right]$ & $N=10^{19}\left[\mathrm{~m}^{-3}\right]$ \\
\hline $\begin{array}{l}\text { Mass density: } \\
\rho=N \cdot 25 m_{p}\left[\mathrm{~kg} \cdot \mathrm{m}^{-3}\right]\end{array}$ & $\rho=4.175 \times 10^{-7}$ & $\rho=4.175 \times 10^{-7}$ \\
$\begin{array}{l}\text { Gradient of Coriolis force: } \\
\beta=2 \Omega_{0} \cos (\lambda) / R\left[\mathrm{~m}^{-1} \mathrm{~s}^{-1}\right]\end{array}$ & $\beta=2.71 \times 10^{-12}$ & $\beta=1.54 \times 10^{-11}$ \\
$\begin{array}{l}\text { Geomagnetic field: } \\
\beta_{B}=-2 B_{\text {eq }} \cos (\lambda) / R[\mathrm{~T} / \mathrm{m}]\end{array}$ & $\beta_{B}=-2.71 \times 10^{-12}$ & $\beta_{B}=-1.54 \times 10^{-11}$ \\
$\begin{array}{l}\text { Rossby speed: } \\
c_{B}=\beta_{B} /\left(\text { en } \mu_{0}\right)[\mathrm{m} / \mathrm{s}]\end{array}$ & $c_{B}=-135$ & $c_{B}=-765$ \\
\hline
\end{tabular}

$(\lambda, n, N)$ are $\left(\lambda=80\left[^{\circ}\right], n=10^{11}\left[\mathrm{~m}^{-3}\right], N=10^{19}\left[\mathrm{~m}^{-3}\right]\right)$, which roughly corresponds to Norway, are given by the second column of Table III. The dispersion relation has a similar character compared to the other cases. However, the wave frequency is lower owing to the smaller $\beta$ value from the gradient of the Coriolis force at high latitude. The lower wave frequency makes the trapping of the waves in the vortex structure occur at a smaller vortex amplitude. The nonlinear simulation shows the dipole structure of the stream function moves imperceptibly during this time interval while the dipole structure of the magnetic fluctuations moves to the west with the speed of $144 \mathrm{~m} / \mathrm{s}(520 \mathrm{~km} / \mathrm{h})$. The value of the Rossby speed is also close to the one which the linear calculation gives. The eigenvectors calculated from the eigenvalues in Eq. (9) show that the magnetic fluctuation for the $\omega_{+}$ roots are substantial, owing to the speed of propagation being just faster than $\left|c_{B}\right|$, while the magnetic fluctuation in the $\omega_{-}$ Rossby wave root is small.

Figure 4 shows the profiles of the structure of magnetic fluctuations for (a) high latitude, $\lambda=80^{\circ}$, (b) middle latitude, $\lambda=45^{\circ}$, and (c) low latitude, $\lambda=10^{\circ}$ for several time slices. From Fig. 4 one sees that at high latitude the structures in the magnetic perturbation move less than $100 \mathrm{~km}$ in $30 \mathrm{~min}$ while at the lowest latitude the magnetic structures moves significantly, $500 \mathrm{~km}$ to the west in $30 \mathrm{~min}$ owing to the stronger gradient of the vertical component of the geomagnetic field. On the scale of this figure the stream function moves imperceptibly for all latitudes. The propagation is governed by the value of $c_{B}$ which is proportional to the value of $\cos (\lambda)$. The charge density $n=10^{11}\left[\mathrm{~m}^{-3}\right]$ corresponds to slow Rossby-Khantadze speed $c_{B}$ of the day-time
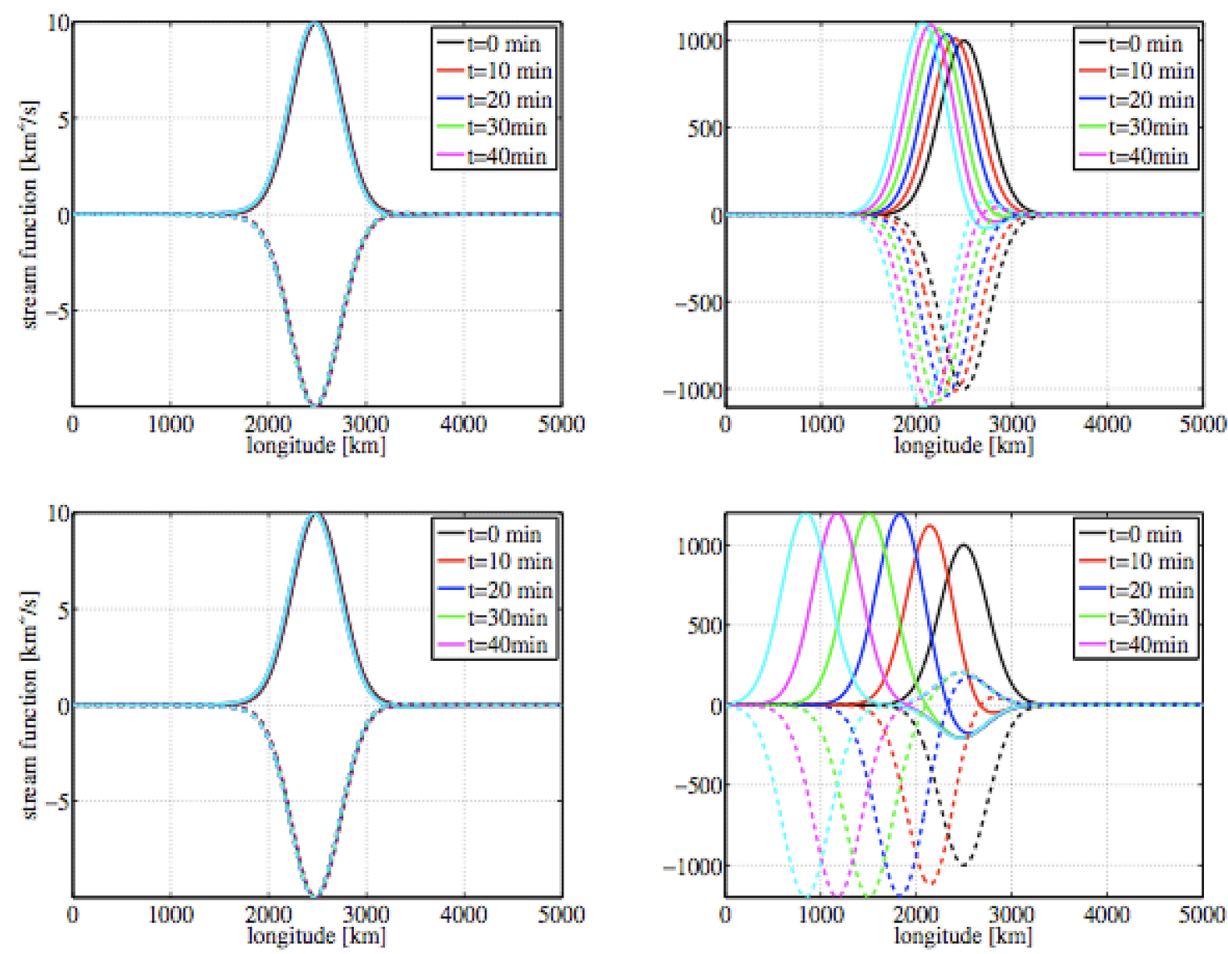

FIG. 4. The cross section of the dipole structures (top panel: high latitude, $\lambda=80^{\circ}$; middle panel: middle latitude, $\lambda=45^{\circ}$; bottom panel: low latitude, $\lambda=10^{\circ}$ ). Solid lines are the horizontal cut of the positive part of the dipole structure, and the dashed lines are the cut of the negative part of the dipole structure. The structure of the stream function moves imperceptibly while the lower latitude has a faster propagation of the waves of magnetic fluctuations owing to the stronger gradient of the vertical geomagnetic field.
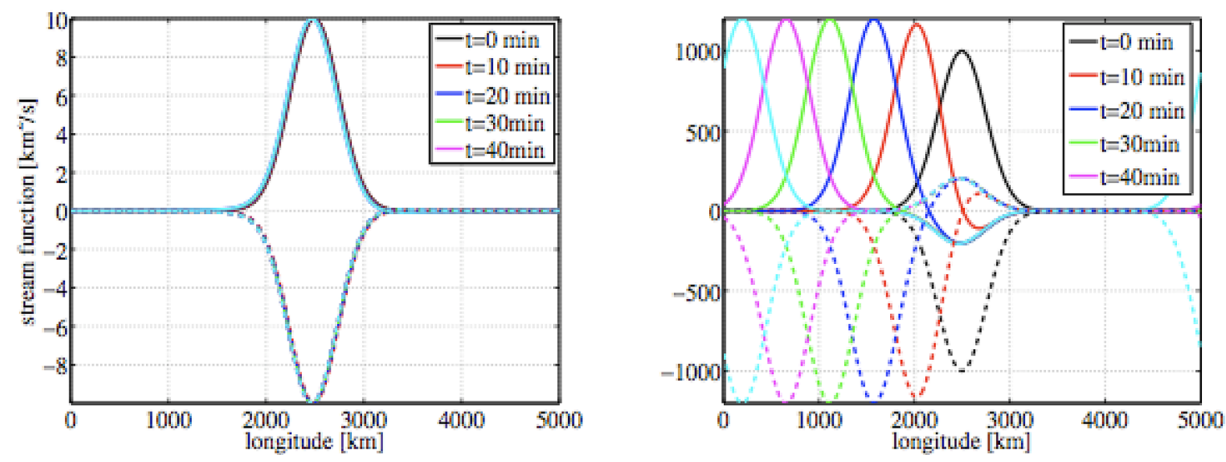
E-layer while the charge density $n=10^{10}\left[\mathrm{~m}^{-3}\right]$ corresponds to fast Rossby-Khantadze speed $c_{B}$ of the night-time ionosphere. The Rossby-Khantadze speed $c_{B}$ varies inversely with the electron density. The parameters of $(\lambda, n, N)$ are $\left(\lambda=45\left[^{\circ}\right], n=10^{10}\left[\mathrm{~m}^{-3}\right], N=10^{19}\left[\mathrm{~m}^{-3}\right]\right)$, and they are given by the third column of Table III. $c_{B}$ becomes 10 times higher in this case than the case of $n=10^{11}\left[\mathrm{~m}^{-3}\right]$. The nonlinear simulation shows the dipole structure of the stream function which hardly moves in this time interval while the dipole structure of the magnetic fluctuations moves to the west with the speed of $5500 \mathrm{~m} / \mathrm{s}(19800 \mathrm{~km} / \mathrm{h})$. The observed propagation speed of the nonlinear solitary dipole structure agrees with the speed derived from the linear modes.

Figure 5 shows the stream function and the magnetic fluctuations at $t=600 \mathrm{~h}$. The continuation of Fig. 3 is shown in the first row of Fig. 5. The energy in these simulation is well conserved and the energy loss is less than $0.0006 \%$ after $\mathrm{t}=600 \mathrm{~h}$. Even after $600 \mathrm{~h}$, we find that the dipole structure retains its solitary vortex character because the amplitude of the dipole satisfies the wave trapping condition. The second row of Fig. 5 shows the typical Rossby wave which is reduced from Eqs. (1) and (2), by set the parameters $\beta_{B}=c_{B}=0$. In the system which has been reduced to be pure Rossby waves, the magnetic fluctuation $b$ behaves as a passive tracer. The simulation shows that the scalar field propagates similar to the dipole structure of the stream function. In the third row of Fig. 5, the nonlinear terms in Eqs. (1) and (2) have been turned off, and the linear system has been solved. As shown in Fig. 5, i.e., linear simulation of the electromagnetic Rossby waves shows the radiative dispersion of the coherent structure into many elongated structures in east-west direction. The dynamics shown in Fig. 5 shows that the simulations which are launched with the large amplitude of the dipole structure are a strong nonlinear state which generates the dipole structure as the solution of the system. Comparison of Figs. 2 and 5 indicate the condition that the
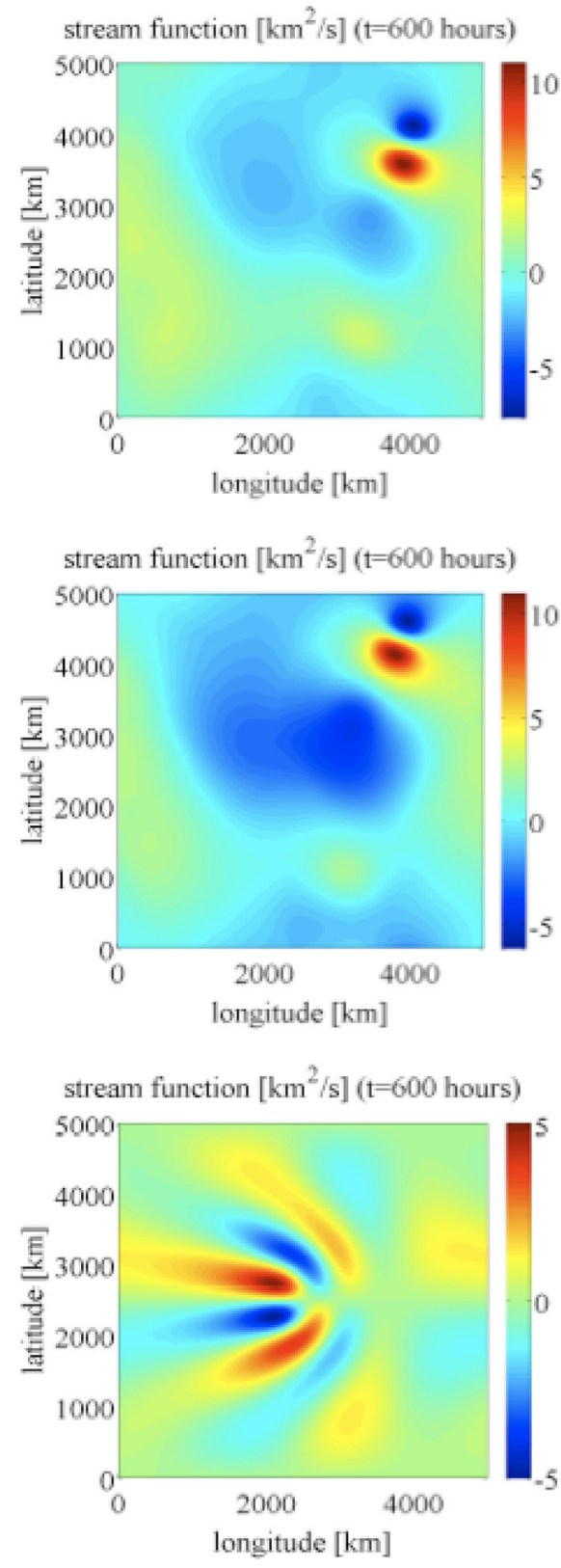
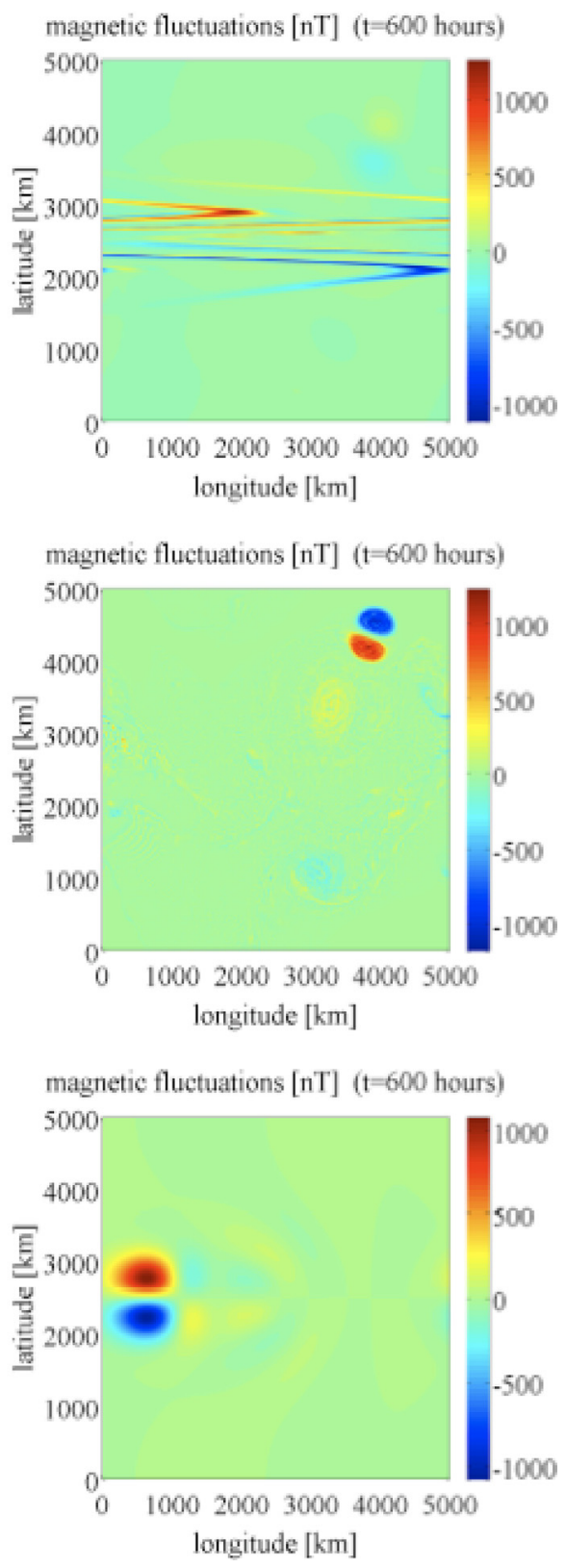

FIG. 5. The stream function and the magnetic fluctuations at $\mathrm{t}=600 \mathrm{~h}$. The continuation of Fig. 3 reaches the first row of Fig. 5. The classical Rossby wave which the magnetic fluctuation is turned off is shown in the second row of Fig. 5. In the system which has been reduced to be pure Rossby waves, the magnetic fluctuation $b$ behaves as a passive tracer. The snapshots of stream function show the existence of a nonlinear dipole structure as a solution of solitary vortex. Concerning to the third row of Fig. 5, the nonlinear terms in Eqs. (1) and (2) have been switched off. As shown in the third row of Fig. 5 , the linear simulation of the electromagnetic Rossby waves shows that the character of the structure is changed, and the structure is forming the radiative dispersive waves radiated into many elongated structures in east-west direction after the convective nonlinearity is turned off. 
dipole structure behaves as a solitary vortex. The nonlinearity which satisfies the wave trapping condition grows even stronger by the $\mathrm{t}=600 \mathrm{~h}$. The group velocity in the $y$-direction of the Rossby wave is replaced by a slightly faster nonlinear solitary wave velocity for the finite amplitude vortices. In the strong nonlinear regime, the effects of the linear components become very small, and there is no well defined channel for keeping the structure in the latitude. For the initial condition used here in Eq. (10), there is a dominant nonlinear dipole vortex and small component of waves. The exact nonlinear vortex structure is complicated and given by Bessel functions in Ref. 12. In the collisions of the approximate vortex solitary structure with the residual waves, the vortex is deflected northward and propagates with the residual northward group velocity which is small and proportional to $\left\langle k_{x} k_{y}\right\rangle$ averaged over the linear mode spectra of the localized structure. Regarding to the magnetic perturbations in the first row of Fig. 5, what is happening to make the streaks of the magnetic perturbations is at some earlier time some wave or nonsoliton fields of magnetic perturbations $b$ are generated and the $b$ is passively convected basically along lines of $x=x_{0}-\left|c_{B}\right| t, y=y_{0}$. As the consequence, there are streaks of the magnetic perturbations left at $\mathrm{t}=600 \mathrm{~h}$.

\section{Couplings between the stream function and the magnetic fluctuations}

As the system of Eqs. (1) and (2) are coupled, there is an interaction between the stream function for the neutral gas velocity and the magnetic fluctuations. Figure 6 shows the snapshots of the stream function (left panels) and the magnetic fluctuations (right panels). The initial condition of the stream function has been put as zero, while the initial condition of the magnetic fluctuations is set to the dipole structure. As time advances, the stream function is generated by the structure of the magnetic fluctuations. What is interesting here is that the movement of the magnetic fluctuations creates a new neutral gas flow structure. The stream function is
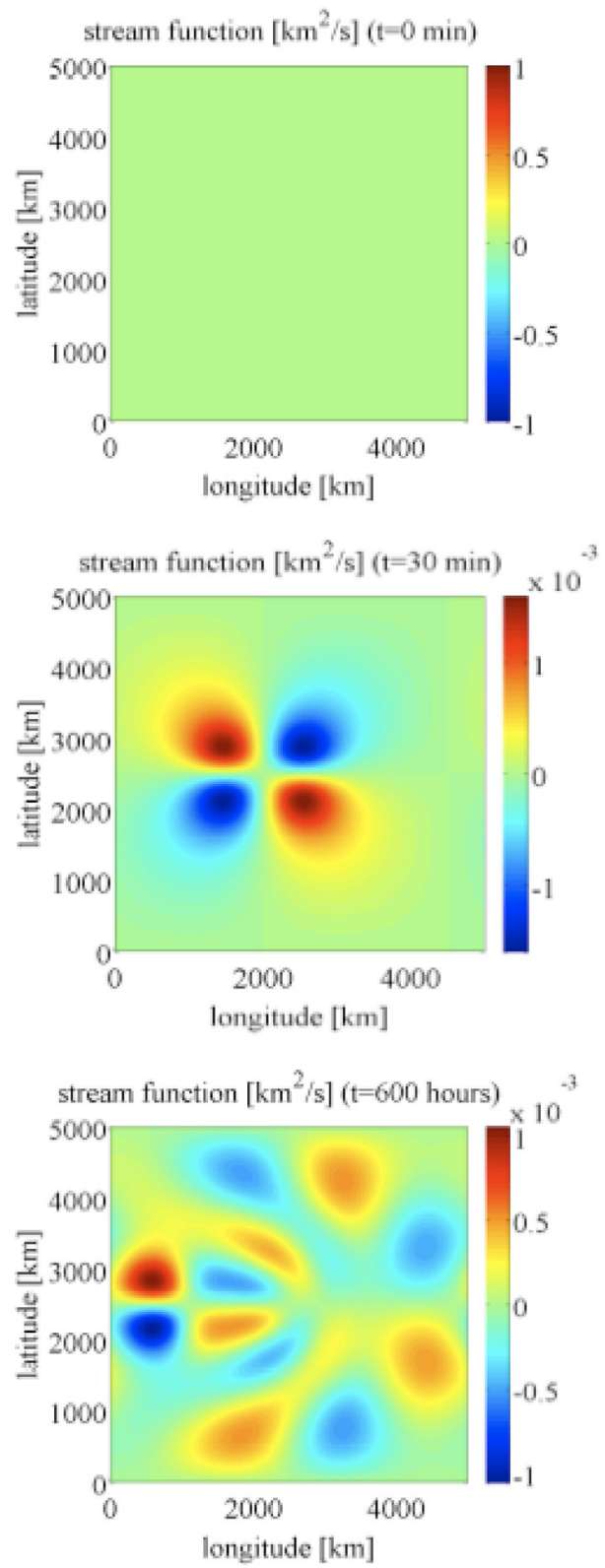
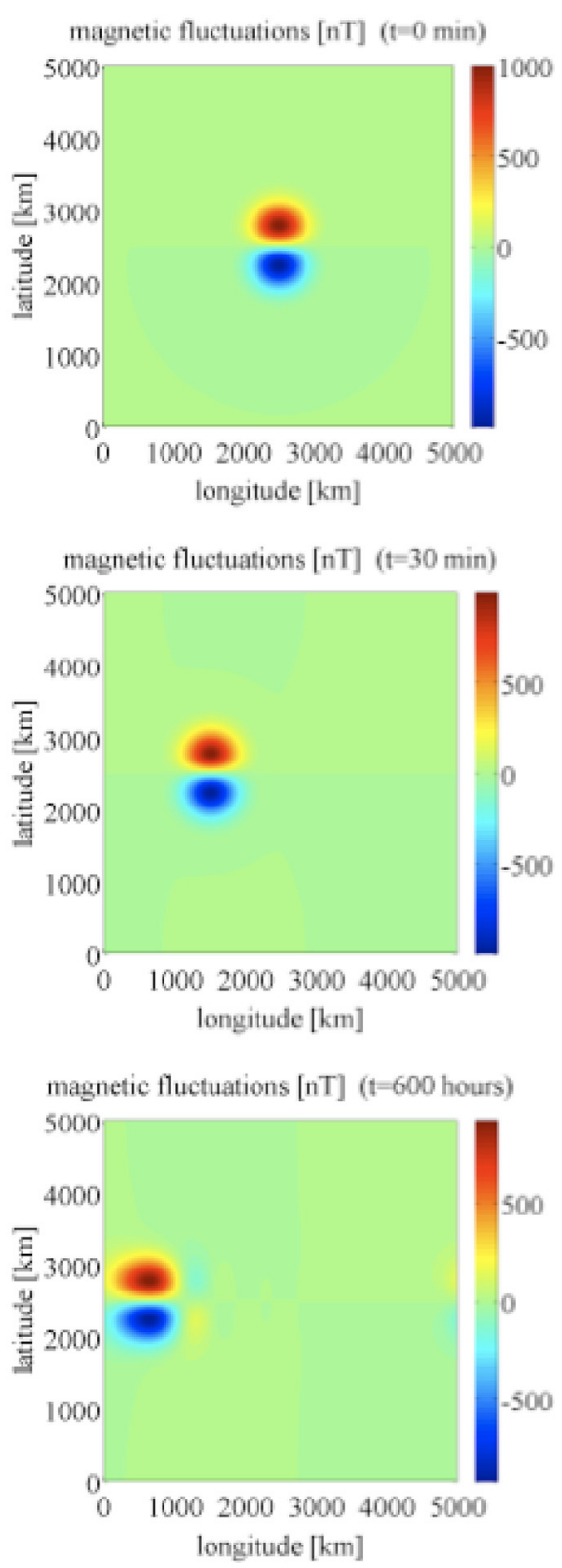

FIG. 6. Snapshot of the stream function (left column) and the magnetic fluctuations (right column) at the initial condition (top rows), $30 \mathrm{~min}$ (middle rows) and $600 \mathrm{~h}$ (bottom rows). What is interesting here is the rapid change in the structure of the stream function. The stream function which is generated by the rapidly propagating magnetic dipole structure (in the right column) has a quadrupole structure. We find that later in time the quadrupole structure of the stream function splits into two dipoles (shown in movie on the website. One stream function dipole structures propagates from east to west direction with the magnetic dipole and the other dipole structures moves imperceptibly as a Rossby vortex remaining at the location where it is generated. 
generated by the magnetic fluctuations forming the quadrupole structure after $30 \mathrm{~min}$. Figure 7 shows the profiles of the stream function and the magnetic fluctuations for several time slices. The position of the structure moves westward according to the propagation of the structure of the magnetic fluctuations. We observe that the quadrupole structure splits into two dipoles in later time slices. The magnetic dipole structures will propagate rapidly from east to west while the Rossby wave dipole structure remains at the location where it is generated for time periods of the order of hours. The stream function which is generated by the magnetic fluctuations is radiated away into many small waves in later time. Just after the generation of the stream function, its amplitude is small. Therefore, the structure of the stream function does not satisfy the wave trapping condition and the behavior is similar with the study of a "small amplitude vortex structure." However, the dipole structure of the magnetic fluctuations keeps the solitary behavior as it satisfies the wave trapping condition.
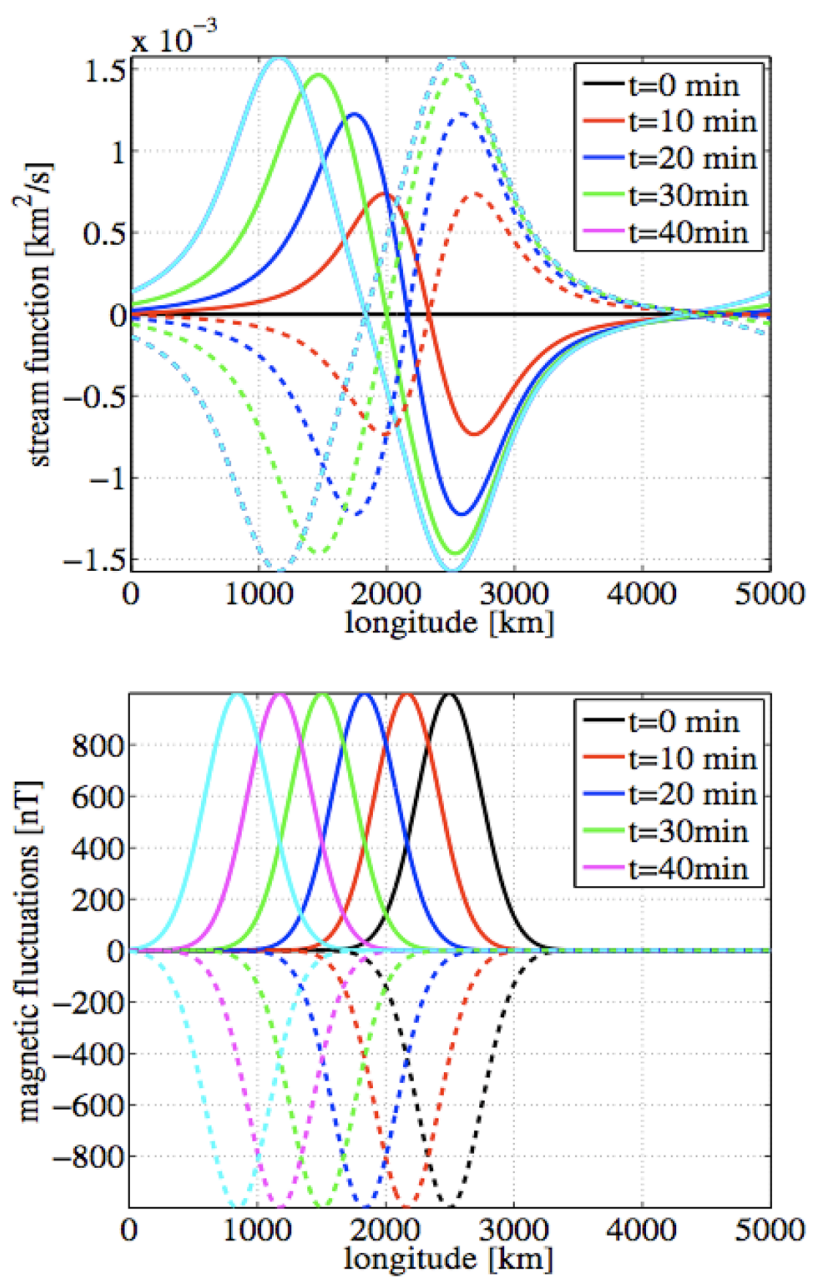

FIG. 7. The profiles of the stream function and the magnetic fluctuations for several time slices. Solid lines are the horizontal cut of the positive part of the dipole structure, and the dashed lines are the cut of the negative part of the dipole structure. The stream function is generated by the magnetic fluctuations, forming the quadrupole structure. The position of the structure moves according to the propagation of the structure the magnetic fluctuations. Note that the quadrupole structure of the stream function will split into two dipoles in later time slices. One dipole structures will propagate from east to west direction with the speeds of the Khantadze and Rossby structures.
Next, the generation of the magnetic fluctuations by the stream function has been investigated. Figure 8 shows the snapshot of the stream function (left panel) and the magnetic fluctuations (right panel). The magnetic fluctuations are formed by the dipole structure of the stream function. Figure 9 shows the profiles of the stream function and the magnetic fluctuations for several time slices. The magnetic fluctuations are generated by the stream function. During the generation of the magnetic fluctuations, the structure of the magnetic fluctuations propagates from east to west. The magnetic fluctuation which is generated by the stream function behaves nonlinearly, i.e., it forms the dipole structure. As to the dynamics of the magnetic fluctuations, just after the generation of magnetic fluctuations its amplitude is small, and it should be radiated away into many waves. However, the stream function keeps the solitary behavior as it has enough amplitude to be a soliton. In these structures the kinetic energy in the flow of the neutral fluid dominates the magnetic energy in Eq. (7) by a large factor. As a consequence, the behavior of magnetic fluctuations is dominated by the dynamics of the stream function, and, finally, the magnetic structure displays a nonlinear dynamics, i.e., it forms a dipole structure.

\section{CONCLUSION}

We presented linear and nonlinear simulations of the coupled system of the fast and slow waves in the E-layer that
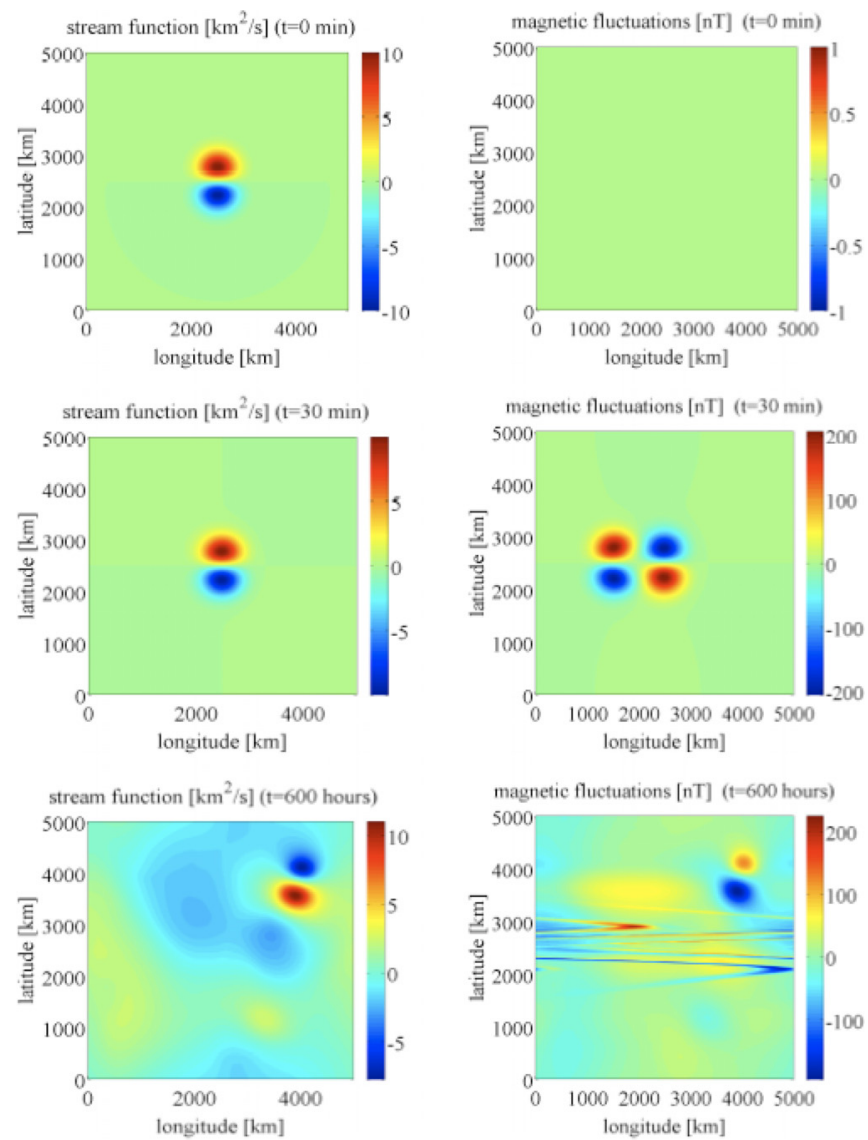

FIG. 8. Snapshot of the stream function (left column) and the magnetic fluctuations (right column) at the initial condition (top rows), $30 \mathrm{~min}$ (middle rows) and $600 \mathrm{~h}$ (bottom rows). The magnetic structure is generated by the stream function, with forming the quadrupole structure. 

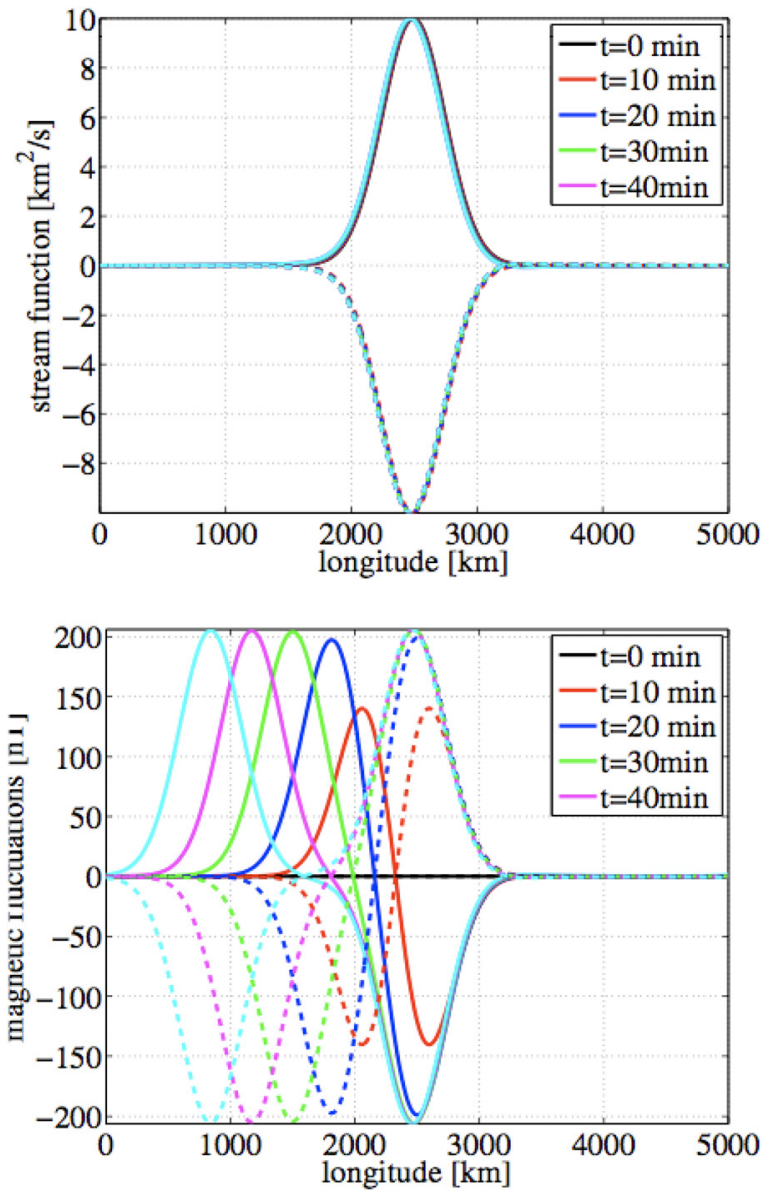

FIG. 9. The profiles of the stream function and the magnetic fluctuations for several time slices. The magnetic fluctuations are generated by the stream function. During the generation of the magnetic fluctuations, the structure of the magnetic fluctuations propagates from east to west while the stream function which generates the magnetic fluctuation moves imperceptibly. Note that the quadrupole structure of the magnetic fluctuations split into two dipoles in later times.

result from the coupling of Rossby wave dynamics of the neutral gas component with the magnetic waves of the weakly ionized plasma component of the gas. The nonlinear simulation show that initial disturbances with significant amplitudes will propagate as coherent vortex structures while small amplitude initial perturbations will radiate a spectrum of waves. The energy of waves is composed of the kinetic energy of the neutral gas and the magnetic energy of the perturbed magnetic field created by the Hall currents. The behavior of the waves and the coherent E-layer structures are shown for several latitudes and initial conditions. The simulations provide a deeper understanding of the theoretical considerations given in Kaladze et al. ${ }^{4}$ and Horton et al. ${ }^{13}$ The two coupled nonlinear partial differential equations for vorticity of the neutral gas with the Lorentz force term from the perturbation of the geomagnetic field produced by the Hall currents give a complex dynamical system that is solved numerically on a large domain of $5000 \mathrm{~km} \times 5000 \mathrm{~km}$ for long time periods up to 25 days. We show that in the large space-scale long-time dynamics in the E-layer there are two components to the dynamics: (1) a form of magnetized Rossby waves that propagates with speeds of the order of $10-20 \mathrm{~m} / \mathrm{s}$ and (2) a fast magnetic disturbance that carries a neutral fluid disturbance with it, owing to the electromagnetic force acting through the ion-neutral drag force. This magnetic disturbance is the Khantadze wave propagating at speeds of the order of $500-1000 \mathrm{~m} / \mathrm{s}$ westward. The total energy in either type of disturbance is conserved in the model equations. In this model the flow kinetic energy and the magnetic energy produced from the electron Hall current (from the $E \times B$ currents) are dissipationless. In the RossbyKhantadze electromagnetic planetary waves, the propagation speed of the solitary waves is of the same order as the speed which is obtained by the linear modes. In future work, we will add driving forces and dissipation to study the corresponding problems of spectral cascades in driven-damped two dimensional turbulence. We will also study how externally applied sheared flows in the neutral gas, spit off one component of the dipole vortex, as well known from neutral gas in geophysical dynamics. ${ }^{1}$

The simulations show for a variety of initial conditions, that there is a slow, coherent Rossby wave structure similar to those occurring in the lower altitude atmosphere and a fast magnetic structure that propagates out from a generic initial Rossby wave perturbation. The magnetic component is particularly important owning to the ability to detect weak magnetic signals in spacecraft magnetometers. Both wave components of the system propagate westward relative to the surface of the Earth. The results for variation with latitude show that low latitude structures propagate faster than the high latitude structures. At low latitudes the simulations need to be expanded to include a global coordinate system since the tangent plane approximation used here is breaking down.

These large scale E-layer structures are important for interpreting the low frequency magnetic pulsations observed as precursors to, and during, large tectonic plate movements, volcano eruptions, explosions, or large impulses to the magnetosphere from solar magnetic storms where high pressure supersonic flows compress the magnetopause.

\section{ACKNOWLEDGMENTS}

S.F. acknowledges the financial support from the French Research Agency (ANR), project SiCoMHD, contract ANR11-BLAN-045. W.H. was supported by NSF Grant 0964692 to the University of Texas at Austin, the University of Aix-Marseille/CNRS, and by IMéRA - Institute for Advanced Study of Aix-Marseille University. This research was partially supported by the International Space Science Institute in Bern (Switzerland) through the grant "Large-scale vortices and zonal winds in planetary atmospheres/ionospheres: Theory vs observations."

${ }^{1}$ A. E. Gill, Atmosphere-Ocean Dynamics (Academic Press, New York, 1982), pp. 232-313.

${ }^{2}$ A. G. Khantadze, V. G. Jandieri, and G. V. Jandieri, Plasma Phys. Rep. 34, 480 (2008).

${ }^{3}$ T. D. Kaladze and W. Horton, Plasma Phys. Rep. 32, 996 (2006).

${ }^{4}$ T. D. Kaladze, G. D. Aburjania, O. A. Kharshiladze, W. Horton, and Y.-H. Kim, "Theory of magnetized Rossby waves in the ionospheric E-layer," J. Geophys. Res. 109, A05302, doi:10.1029/2003JA010049 (2004).

${ }^{5}$ T. D. Kaladze, L. V. Tsamalashvili, and I. Z. Kahlon, J. Plasma Phys. 77, 813 (2011). 
${ }^{6}$ D. J. Cavalieri, R. J. Deland, J. F. Poterna, and R. F. Gavin, J. Atmos. Terr. Phys. 36, 561 (1974).

${ }^{7}$ V. P. Burmaka, L. S. Kostrov, and L. F. Chernogor, "Statistical characteristics of doppler HF radar signals during sounding the middle ionosphere disturbed by rocket launches and solar terminator," Radiofiz. Radioastron. 8, 143 (2003).

${ }^{8}$ P. R. Fagundes, V. G. Pillat, M. J. Bolzan, Y. Sahai, F. Becker-Guedes, J. R. Abalde, S. L. Aranha, and J. A. Bittencourt, J. Geophys. Res. 110, A12302, doi:10.1029/2005JA011115 (2005).
${ }^{9}$ L. S. Alperovich and E. N. Fedorov, Hydrodynamic Waves in the Magnetosphere and the Ionosphere (Springer, 2007).

${ }^{10}$ A. R. Lawrence and M. J. Jarvis, J. Atmos. Sol.-Terr. Phys. 65, 765 (2003). ${ }^{11}$ T. D. Kaladze, L. Z. Kahlon, and L. V. Tsamalashvili, Phys. Plasmas 19, 022902 (2012)

${ }^{12}$ W. Horton and Y.-H. Ichikawa, Chaos and Structures in Nonlinear Plasmas (World Scientific Press, 1996).

${ }^{13}$ W. Horton and A. Hasegawa, Chaos 4, 227 (1994).

${ }^{14}$ A. Arakawa, J. Comput. Phys. 1, 119 (1966). 